\title{
STUDY ON THE CONVERGENCE OF THE GENERALIZED DECISION FEEDBACK EQUALIZER ALGORITHM WITH ERROR FEEDBACK FILTER
}

\author{
Jong Sam Lee ${ }^{1}$ and Yong Chan Lee ${ }^{2}$ \\ ${ }^{1}$ Faculty of Communications, Kim Chaek University of Technology, Democratic People's Republic of Korea \\ ${ }^{2}$ College of Information Engineering, Wonsan Jogunsil University of technology, Democratic People's Republic of Korea
}

\begin{abstract}
In digital communications, a time-dispersive variation channel causes inter-symbol interference (ISI) and distorts the received signal, thus degrading the system performance. The distortion caused by ISI may be mitigated by passing the received signal through an adaptive equalizer such as linear equalizer, maximum likelihood sequence estimator (MLSE) and decision feedback equalizer (DFE). This paper demonstrates a new approach to improving the convergence of the DFE algorithm with error feedback filter in order to reduce the correlation of error signals and also to decrease the residual error variance. The paper theoretically analyzes the convergence of generalized DFE with error feedback filter, and proves that the minimum mean square error (MMSE) monotonically decreases and stably converges when the order of the error feedback filter increases. The simulation clearly shows that the proposed DFE algorithm results in better ERLE (Echo Return Loss Enhancement) than previous ones and particularly the significant improvement of BER performance in case that the number of taps in the error feedback filter increases under low SNR environment.
\end{abstract}

Keywords:

Decision Feedback Equalizer, Error Feedback Filter, ISI, Convergence, MMSE

\section{INTRODUCTION}

Considerable effort has been devoted to the research on data transmission systems that utilize efficiently the available channel bandwidth. The digital data transmission via linear channel is affected by ISI and thermal noise. To reduce the impact of ISI due to multipath channels, adaptive equalization is commonly used.

There are mainly two adaptive equalization algorithms: maximum likelihood sequence estimator (MLSE) [2-5] and decision feedback equalizer (DFE) [6-8]. In general, MLSE can yield better performance than DFE; however, it is suffering from higher computational complexity. DFE is well known for its simple structure, which provides satisfactory tradeoff between cost effectiveness and performance [1, 4]. From the comparison between MLSE and DFE, this paper considers DFE algorithm which is a nonlinear equalization algorithm.

DFE basically consists of a feedforward and a feedback sections with transversal filters in which taps are spaced at the reciprocal of the symbol rate. Since the channel is time-varying, the taps of DFE are commonly adjusted by some adaptive algorithms such as Least Mean Square (LMS) and Recursive Least Square (RLS). RLS algorithm has a fast convergence property, however it requires computational complexity. By contrast, LMS algorithm has strengths of simplicity and robustness even though it has a slow convergence [2]. Therefore, it becomes the consideration in this paper.
Since DFE has its recursive structure, it tends to suffer from error propagation, thus resulting in mean square error (MSE) degradation [9]. Many techniques have been proposed to reduce the error propagation and thus to improve the overall DFE performance. In [10], researchers addressed the problem of the error propagation as a result of both input of errors in the feedback filter and the divergence due to "false errors" in the least mean square decision directed algorithm (LMS-DD). In fact, it is difficult to distinguish between the error propagations due to the former and the latter. To tackle this problem, the preceding researchers proposed a weighted decision feedback equalizer (WDFE) which addresses both the aspects and offers the advantage of limiting the error propagation phenomenon [11]. Meanwhile, error feedback technique is a general method to reduce the error signal existing inherently in quantization process [12] and widely used in applications such as predictive coding [13], [14]. Based on this principle, Wen-Jiang [15] proposed a DFE structure with one-tap error feedback filter and proved that it produced better convergence performance than the previous DFEs. However, he restricted the number of taps to one and analyzed theoretically the convergence performance. Overall, the researchers in [9]-[15] tended to decrease the error propagation caused by the adaptive filter processing or the correlation existing in the error signal. Besides, the study is proposed for improving the performance of wideband quadrature amplitude modulation (QAM) receiver by changing the structure of DFE [16]. This paper proposed a new DEF structure of which the feedback filter preceded the forward filter, applied to baseband high order QAM demodulator with different received antennas and ensured its performance.

The main aim of this paper is to generalize the DFE with error feedback filter by analyzing convergence performance and convergence stability according to the increase of the number of taps in error feedback filter. The rest of this paper is organized as follows: The convergence of the generalized DFE is theoretically analyzed in section 2 and its convergence stability according to the number of taps in error feedback filter is proved in section 3 . Section 4 presents simulation results, followed by conclusion in section 5 .

\section{CONVERGENCE OF THE GENERALIZED DFE WITH ERROR FEEDBACK FILTER}

\subsection{CONVERGENCE OF THE CONVENTIONAL DFE}

The Fig. 1 shows the conventional DFE system which consists of a Feed-Forward (FF) filter and a Feed-Back (FB) filter. 


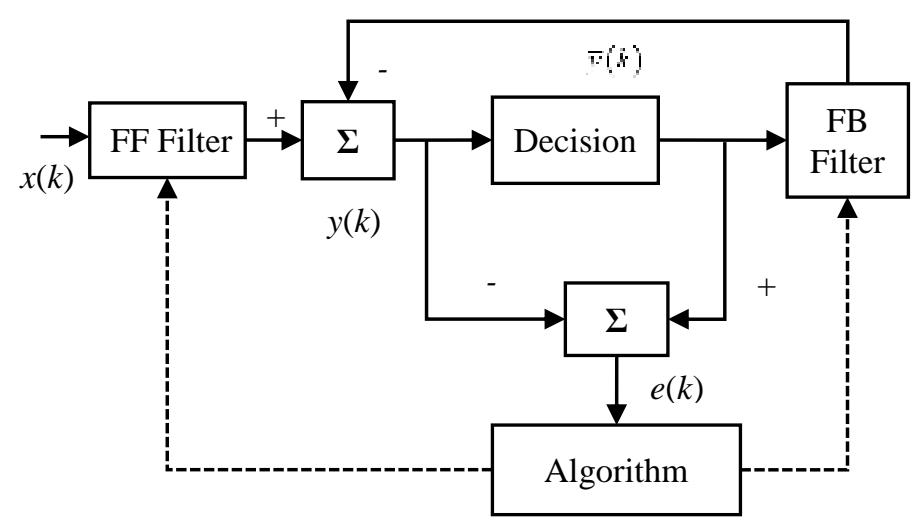

Fig.1. Conventional Decision Feedback Equalizer

As compared to Linear Equalizer (LE), the DFE operates to reduce ISI by using the FB output data from the decision part through the FB filter.

The input vector $\mathbf{U}(k)$ and filter weight vector $\mathbf{W}(k)$ at instant $k$ is given as follows

$$
\begin{aligned}
\mathbf{U}(k) & =[x(k), x(k-1), \ldots, x(k-N), \bar{y}(k-1), \bar{y}(k-2), \ldots, \bar{y}(k-M)] \\
\mathbf{W}(k) & =\left[w_{f, 0}(k), w_{f, 1}(k), \cdots, w_{f, N}(k), w_{b, 1}(k), w_{b, 2}(k), \cdots, w_{b, M}(k)\right]
\end{aligned}
$$

where the number of taps in the feedforward filter and the number in the decision feedback filter are $N+1$ and $M$, respectively. And $x(k)$ and $\bar{y}(k)$ denote the input signal and the decision value of the filter output respectively. The output of the DFE is given as Eq.(3),

$$
\begin{aligned}
y(k) & =\sum_{i=0}^{N} w_{f, i}(k) \cdot x(k-i)+\sum_{j=1}^{M} w_{b, j}(k) \cdot \bar{y}(k-j) \\
& =\mathbf{U}^{T}(k) \mathbf{W}(k)=\mathbf{W}^{T}(k) \mathbf{U}(k) .
\end{aligned}
$$

Accordingly, the error signal $e(k)$ is calculated by Eq.(4),

$$
e(k)=d(k)-y(k)
$$

The cost function [2] is defined as the mean square error (MSE) calculated by,

$$
J=E\left[e^{2}(k)\right]=E\left[d^{2}(k)\right]-2 \mathbf{P}^{T} \mathbf{W}+\mathbf{W}^{T} \mathbf{R W}
$$

where $E[\cdot]$ means the statistical expectation. The crosscorrelation vector $\mathbf{P}$ and auto-correlation matrix $\mathbf{R}$ are given as follows,

$$
\begin{aligned}
& \mathbf{P}=E[d(k) \cdot \mathbf{U}(k)] \\
& \mathbf{R}=E\left[\mathbf{U}^{T}(k) \cdot \mathbf{U}(k)\right]
\end{aligned}
$$

The optimal weight vector $\mathbf{W}^{*}$ is then obtained by,

$$
\mathbf{W}^{*}=\mathbf{R}^{-1} \mathbf{P}
$$

Finally, the minimum MSE (MMSE) is obtained by substituting $\mathbf{W}^{*}$ to Eq.(5).

$$
J_{\min }=E\left[d^{2}(k)\right]-\mathbf{P}^{T} \cdot \mathbf{R}^{-1} \cdot \mathbf{P}
$$

\subsection{CONVERGENCE OF THE GENERALIZED DFE WITH ERROR FEEDBACK FILTER}

The conventional DFE is not possible to remove completely the correlation of noise samples by a linear predictor composed of a finite number of taps. Therefore, the DFE cannot be optimal in case that the noise at the slicer input is not white.

In this paper, the generalized DFE algorithm with L-taps error feedback filter which is considered as an extension of the DFE with one-tap error feedback filter [15]. Fig.2.

The block diagram of the proposed approach is shown in

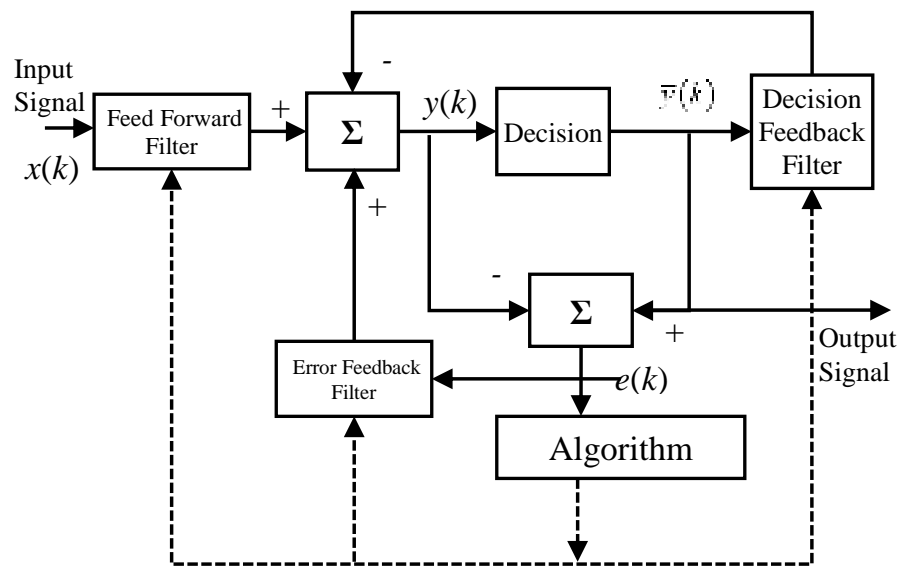

Fig.2. Decision Feedback equalizer with L-taps error feedback filter

The proposed DFE consists of the conventional DFE and an error feedback filter. In Fig.2, the input vector is defined as $\overrightarrow{\mathbf{U}}(k)$, and the filter weight vector as $\overline{\mathbf{W}}(k)$ at instant $k$.

$$
\begin{gathered}
\overline{\mathbf{U}}(k)=[x(k), x(k-1), \ldots, x(k-N), \bar{y}(k-1), \bar{y}(k-2), \ldots \\
\ldots, \bar{y}(k-M), e(k-1), e(k-2), \ldots, e(k-L)] \\
\overline{\mathbf{W}}(k)=\left[w_{f, 0}(k), w_{f, 1}(k), \ldots, w_{f, N}(k), w_{b, 1}(k), w_{b, 2}(k), \ldots\right. \\
\left.\cdots, w_{b, M}(k), w_{e, 1}(k), w_{e, 2}(k), \ldots, w_{e, L}(k)\right]
\end{gathered}
$$

where the numbers of taps in the feedforward filter, the decision feedback filter and the error feedback filter are $N+1, M$ and $L$, respectively.

The total output is given as

$$
\begin{gathered}
y(k)=\overline{\mathbf{W}}^{T}(k) \overline{\mathbf{U}}(k)=\sum_{i=0}^{N} w_{f, i}(k) \cdot x(k-i)+ \\
+\sum_{j=1}^{M} w_{b, j}(k) \cdot \bar{y}(k-j)+\sum_{l=1}^{L} w_{e, l}(k) \cdot e(k-l)
\end{gathered}
$$

The error signal is expressed as

$$
\bar{e}(k)=d(k)-y(k)=d(k)-\overline{\mathbf{W}}^{T}(k) \overline{\mathbf{U}}(k)
$$

For the generalized DFE with L-taps error feedback filter, the cost function is also defined as MSE. 


$$
\begin{aligned}
\bar{J}=E\left[\bar{e}^{2}(k)\right]=E[ & \left.d^{2}(k)\right] \\
& -2 \cdot \overline{\mathbf{W}}^{T}(k) \cdot \mathbf{P}+\overline{\mathbf{W}}^{T}(k) \cdot \overline{\mathbf{R}} \cdot \overline{\mathbf{W}}(k)
\end{aligned}
$$

The cross-correlation vector $\overline{\mathbf{P}}$ and auto-correlation matrix $\overline{\mathbf{R}}$ are given as follows.

$$
\begin{aligned}
& \overline{\mathbf{P}}=E[d(k) \cdot \overline{\mathbf{U}}(k)] \\
& \overline{\mathbf{R}}=E\left[\overline{\mathbf{U}}^{T}(k) \cdot \overline{\mathbf{U}}(k)\right]
\end{aligned}
$$

where,

$\overline{\mathbf{P}}$ is a $1 \times(N+M+L+1)$ vector and

$\overline{\mathbf{R}}$ is a $(N+M+L+1)$ matrix.

The optimal weight vector $\overline{\mathbf{W}}^{*}$ is then obtained by,

$$
\overline{\mathbf{W}}^{*}=\overline{\mathbf{R}}^{-1} \cdot \overline{\mathbf{P}}
$$

Finally, the minimum MSE is represented by substituting $\overline{\mathbf{W}}^{*}$ to Eq.(14),

$$
\bar{J}_{\text {min }}=E\left[d^{2}(k)\right]-\overline{\mathbf{P}}^{T} \cdot \overline{\mathbf{R}}^{-1} \cdot \overline{\mathbf{P}}
$$

Next, let me compare the proposed approach with the conventional DFE.

Using Eq.(6) and Eq.(7), Eq.(15) and Eq.(16) are rewritten as,

$$
\begin{gathered}
\overline{\mathbf{R}}=\left[\begin{array}{cc}
\mathbf{R} & \mathbf{D} \\
\mathbf{D}^{T} & \mathbf{E r}
\end{array}\right] \\
\overline{\mathbf{P}}=[\mathbf{P}, \mathbf{Q}]
\end{gathered}
$$

where,

$$
\begin{gathered}
\mathbf{Q}=\mathbf{Q}_{L}=E[d(k) \cdot e(k-1), \cdots, d(k) \cdot e(k-L)]= \\
=E[e(k) \cdot[e(k-1), \cdots, e(k-L)]] \\
\mathbf{D}=E[e(k-1) \mathbf{U}(k), e(k-2) \mathbf{U}(k), \ldots, e(k-L) \mathbf{U}(k)] \\
\mathbf{E}_{\mathbf{r}}=E\left[\begin{array}{ccc}
e^{2}(k-1) & \cdots & e(k-1) e(k-L) \\
\vdots & \ddots & \vdots \\
e(k-1) e(k-L) & \cdots & e^{2}(k-L)
\end{array}\right]
\end{gathered}
$$

By using block matrix inverse lemma, $\overline{\mathbf{R}}^{-1}$ can be calculated.

$$
\overline{\mathbf{R}}^{-1}=\left[\begin{array}{ll}
\mathbf{R}^{-1}+\mathbf{R}^{-1} \cdot \mathbf{D} & -\mathbf{R}^{-1} \cdot \mathbf{D} \\
\cdot\left(\mathbf{E r}-\mathbf{D}^{T} \cdot \mathbf{R}^{-1} \cdot \mathbf{D}\right)^{-1} & \left(\begin{array}{l}
\mathbf{E r}-\mathbf{D}^{T} \\
\cdot \mathbf{R}^{-1} \cdot \mathbf{D}
\end{array}\right)^{-1} \\
\cdot \mathbf{D}^{T} \cdot \mathbf{R}^{-1} & \left(\begin{array}{l}
\mathbf{E r}-\mathbf{D}^{T} \\
\cdot \mathbf{R}^{-1} \cdot \mathbf{D}
\end{array}\right)^{-1} \\
-\left(\begin{array}{l}
\mathbf{E r}-\mathbf{D}^{T} \\
\cdot \mathbf{R}^{-1} \cdot \mathbf{D}
\end{array}\right)^{-1} \cdot \mathbf{D}^{T} \cdot \mathbf{R}^{-1} &
\end{array}\right]
$$

Accordingly, the second term on the right-hand side of Eq.(18) can be rewritten as,

$$
\begin{aligned}
& \overline{\mathbf{P}}^{T} \cdot \overline{\mathbf{R}}^{-1} \cdot \overline{\mathbf{P}}=\mathbf{P}^{T} \cdot \mathbf{R}^{-1} \cdot \mathbf{P} \\
& +\left[\begin{array}{l}
\mathbf{P}^{T} \cdot \mathbf{R}^{-1} \cdot \mathbf{D} \cdot\left(\mathbf{E}_{\mathbf{r}}-\mathbf{D}^{T} \cdot \mathbf{R}^{-1} \cdot \mathbf{D}\right)^{-1} \\
-\mathbf{Q}^{T} \cdot\left(\mathbf{E r}-\mathbf{D}^{T} \cdot \mathbf{R}^{-1} \cdot \mathbf{D}\right)^{-1}
\end{array}\right] \cdot\left(\mathbf{D}^{T} \cdot \mathbf{R}^{-1} \cdot \mathbf{D}-\mathbf{Q}\right)
\end{aligned}
$$

Thus, the minimum MSE of the proposed DFE approach is rewritten by substituting Eq.(25) into Eq.(18),

$$
\begin{gathered}
\bar{J}_{\min }=E\left[d^{2}(k)\right]-\mathbf{P}^{T} \cdot \mathbf{R}^{-1} \cdot \mathbf{P}-\left[\begin{array}{l}
\mathbf{P}^{T} \cdot \mathbf{R}^{-1} \cdot \mathbf{D} \cdot\left(\mathbf{E}_{\mathbf{r}}-\mathbf{D}^{T} \cdot \mathbf{R}^{-1} \cdot \mathbf{D}\right)^{-1} \\
-\mathbf{Q}^{T} \cdot\left(\mathbf{E r}-\mathbf{D}^{T} \cdot \mathbf{R}^{-1} \cdot \mathbf{D}\right)^{-1}
\end{array}\right] \\
\cdot\left(\mathbf{D}^{T} \cdot \mathbf{R}^{-1} \cdot \mathbf{D}-\mathbf{Q}\right)
\end{gathered}
$$

Since $J_{\min }=E\left[d^{2}(k)\right]-\mathbf{P}^{T} \cdot \mathbf{R}^{-1} \cdot \mathbf{P}$ is the minimum MSE of the conventional DFE, Eq.(26) can be further simplified as,

$$
\bar{J}_{\text {min }}=J_{\text {min }}-\left[\begin{array}{l}
\mathbf{P}^{T} \cdot \mathbf{R}^{-1} \cdot \mathbf{D} \cdot\left(\mathbf{E}_{\mathbf{r}}-\mathbf{D}^{T} \cdot \mathbf{R}^{-1} \cdot \mathbf{D}\right)^{-1} \\
-\mathbf{Q}^{T} \cdot\left(\mathbf{E r}-\mathbf{D}^{T} \cdot \mathbf{R}^{-1} \cdot \mathbf{D}\right)^{-1}
\end{array}\right]
$$

$$
\cdot\left(\mathbf{D}^{T} \cdot \mathbf{R}^{-1} \cdot \mathbf{D}-\mathbf{Q}\right)
$$

where, the vector D consists of various cross-correlations between the input signal and the error signal in Eq.(22). The error signal vector and the input signal vector are orthogonal $(\mathbf{D}=\mathbf{O})$ when the filter weight vector $\mathbf{W}$ converges to the optimal weight vector $\mathbf{W}^{*}$. Thus, Eq.(27) can be more simplified as

$$
\bar{J}_{\text {min }}=J_{\min }-\mathbf{Q}^{T} \cdot \mathbf{E r}^{-1} \cdot \mathbf{Q}
$$

The second term $\mathbf{Q}^{T} \cdot \mathbf{E} \mathbf{r}^{-1} \cdot \mathbf{Q}$ in Eq.28 is in the second order type and not negative because the assumed positive matrix $\mathbf{E}_{\mathbf{r}}$ results in the positive matrix $\mathbf{E}_{\mathbf{r}}^{-1}$.

$$
\mathbf{Q}^{T} \cdot \mathbf{E r}^{-1} \cdot \mathbf{Q} \geq 0
$$

Hence, the feedback of the error signal reduces the MMSE by $\mathbf{Q}^{T} \cdot \mathbf{E r}^{-1} \cdot \mathbf{Q}$. For $L=1$, Eq.(28) reduces to the Feng Wen-jiang Algorithm [15] and accordingly, the proposed algorithm is referred to as the extended the Feng Wen-jiang Algorithm.

However, it is recommended to decide whether $\mathbf{Q}^{T} \cdot \mathbf{E r}^{-1} \cdot \mathbf{Q}$ monotonically increases when the number of taps $L$ increases. In other words, it should be decided whether the MMSE improves better when the number of taps in the error feedback filter increases. The next section will discuss it.

\section{CONVERGENCE STABILITY OF THE GENERALIZED DFE}

According to Eq.(21), the following parameter is denoted as follows when the order of the error feedback filter is $L+1$.

$$
\mathbf{Q}_{L+1} \stackrel{\text { def }}{=}(\mathbf{Q}, E[e(k), e(k-L-1)])^{T}
$$

The error signals in cases that the numbers of taps are $L$ and $L+1$ are written as follows.

$$
\mathbf{E}_{\mathbf{r}} \stackrel{\text { def }}{=} \mathbf{E}_{\mathbf{r}}^{(L)}=\left[\begin{array}{ccc}
E\left[e^{2}(k-1)\right] & \cdots & E[e(k-1) e(k-L)] \\
\vdots & \ddots & \vdots \\
E[e(k-1) e(k-L)] & \cdots & E\left[e^{2}(k-L)\right]
\end{array}\right]
$$




$$
\mathbf{E}_{\mathbf{r}}^{(L+1)}=\left[\begin{array}{cc}
\mathbf{E}_{\mathbf{r}}^{(L)}, & E[e(k-L-1) \bar{e}(L)] \\
E[e(k-L-1) \bar{e}(L)], & E\left[e^{2}(k-L-1)\right]
\end{array}\right]
$$

Here, let $\alpha(L+1)$ and $\mathbf{V}$ be as follows,

$$
\begin{gathered}
\alpha(L+1)=E\left[e^{2}(k-L-1)\right] \\
\mathbf{V}=E[e(k-L-1) \bar{e}(L)]= \\
=E[e(k-L-1) e(k-1), e(k-L-1) e(k-2), \cdots, e(k-L-1) e(k-L)]
\end{gathered}
$$

Calculating the inverse matrix $\left(\mathbf{E}_{\mathbf{r}}^{(L+1)}\right)^{-1}$ leads to the Eq.(34),

$$
\left(\mathbf{E}_{\mathbf{r}}^{(L+1)}\right)^{-1}=\left[\begin{array}{cc}
\mathbf{E}_{\mathbf{r}}^{-1}+\frac{\mathbf{E}_{\mathbf{r}}^{-1} \mathbf{V}^{T} \mathbf{V} \mathbf{E}_{\mathbf{r}}^{-1}}{S} & -\frac{\mathbf{E}_{\mathbf{r}}^{-1} \mathbf{V}^{T}}{S} \\
-\frac{\mathbf{V E}}{S} & S^{-1}
\end{array}\right]
$$

where $S$ is scalar and $S=\alpha(L+1)-\mathbf{V E}_{\mathbf{r}}^{-1} \mathbf{V}^{T}$.

Therefore, Eq.(30) and Eq.(34) result in the following equation.

$$
\begin{gathered}
\mathbf{Q}_{L+1}^{T}\left(\mathbf{E}_{\mathbf{r}}^{L+1}\right)^{-1} \mathbf{Q}_{L+1}= \\
=\mathbf{Q}^{T} \mathbf{E}_{\mathbf{r}}^{-1} \mathbf{Q}+\frac{\mathbf{Q}^{T} \mathbf{E}_{\mathbf{r}}^{-1} \mathbf{V}^{T} \mathbf{V} \mathbf{E}_{\mathbf{r}}^{-1} \mathbf{Q}}{S}-E[e(k) e(k-L-1)] \frac{\mathbf{V E}_{\mathbf{r}}^{-1} \mathbf{Q}}{S} \\
-\frac{\mathbf{Q}^{T} \mathbf{E}_{\mathbf{r}}^{-1} \mathbf{V}^{T} E[e(k) e(k-L-1)]}{S}+\frac{E^{2}[e(k) e(k-L-1)]}{S}
\end{gathered}
$$

By using $\left(\mathbf{E}_{\mathbf{r}}^{-1}\right)^{T}=\mathbf{E}_{\mathbf{r}}^{-1}$ (because $\left.\left(\mathbf{E}_{\mathbf{r}}\right)^{T}=\mathbf{E}_{\mathbf{r}}\right)$ and $\mathbf{Q}^{T} \mathbf{E}_{\mathbf{r}}^{-1} \mathbf{V}^{T}=$ $\mathbf{V E}_{\mathrm{r}}^{-1} \mathbf{Q}$, the following equation can be obtained.

$$
\left(\mathbf{V E} \mathbf{E}_{\mathbf{r}}^{-1} \mathbf{Q}\right)^{2}=\left(\mathbf{V E} \mathbf{E}_{\mathbf{r}}^{-1} \mathbf{Q}\right)^{T}\left(\mathbf{V E}_{\mathbf{r}}^{-1} \mathbf{Q}\right)=\mathbf{Q}^{T} \mathbf{E}_{\mathbf{r}}^{-1} \mathbf{V}^{T} \mathbf{V} \mathbf{E}_{\mathbf{r}}^{-1} \mathbf{Q}
$$

Therefore Eq.(35) is given as,

$$
\begin{gathered}
\mathbf{Q}_{L+1}^{T}\left(\mathbf{E}_{\mathbf{r}}^{L+1}\right)^{-1} \mathbf{Q}_{L+1}=\mathbf{Q}^{T} \mathbf{E}_{\mathbf{r}}^{-1} \mathbf{Q}+\frac{\left(\mathbf{V} \mathbf{E}_{\mathbf{r}}^{-1} \mathbf{Q}\right)^{2}}{S} \\
-\frac{2 E[e(k) e(k-L-1)] \mathbf{V E}_{\mathbf{r}}^{-1} \mathbf{Q}}{S}+\frac{E^{2}[e(k) e(k-L-1)]}{S}
\end{gathered}
$$

If $\quad \frac{\left(\mathbf{V E}_{\mathbf{r}}^{-1} \mathbf{Q}\right)^{2}}{S}=a \quad$ and $\frac{E^{2}[e(k) e(k-L-1)]}{S}=b$, then $\frac{\left(\mathbf{V E} \mathbf{E}_{\mathbf{r}}^{-1} \mathbf{Q}\right)^{2}}{S}+\frac{E^{2}[e(k) e(k-L-1)]}{S} \geq-2 \mathbf{V E}_{\mathbf{r}}^{-1} \mathbf{Q} \frac{E\left[\begin{array}{l}e(k) \cdot \\ e(k-L-1)\end{array}\right]}{S}$ using the formula $a+b \geq 2 \sqrt{a b}$ in case of $S>0$.

$$
\mathbf{Q}_{L+1}^{T}\left(\mathbf{E}_{\mathbf{r}}^{(L+1)}\right)^{-1} \mathbf{Q}_{L+1} \geq \mathbf{Q}^{T} \mathbf{E}_{\mathbf{r}}^{-1} \mathbf{Q}
$$

That is, Eq.(37) means that the increase of the number of taps is accompanied by the increase of the second term in Eq. 28 when $S>0$.

However, if $S<0$, then $\mathbf{Q}_{L+1}^{T}\left(\mathbf{E}_{\mathbf{r}}^{(L+1)}\right)^{-1} \mathbf{Q}_{L+1} \leq \mathbf{Q}^{T} \mathbf{E}_{\mathbf{r}}^{-1} \mathbf{Q}$.
Thus the signedness of $S$ determines the increase/decrease of the second term in Eq.(28).

The signedness of $S=\alpha_{L+1}-\mathbf{V E}_{\mathbf{r}}^{-1} \mathbf{V}^{T}=E\left[e^{2}(k-L-1)\right]$ $-\mathbf{V E} \mathbf{E}_{\mathbf{r}}^{-1} \mathbf{V}^{T}$ should be decided.

If $\alpha_{L+1}=\lambda(0)$ for generality, then

$$
\mathbf{V}=[\lambda(L), \lambda(L-1), \cdots, \lambda(1)],
$$

where $\lambda(i)=E[e(k-j) e(k-j-i)]$.

In addition, Eq.(32) can be rewritten as follows.

$$
\mathbf{E}_{\mathbf{r}}=\left[\begin{array}{cccc}
\lambda(0) & \lambda(1) & \cdots & \lambda(L-1) \\
\lambda(1) & \lambda(0) & \cdots & \lambda(L-2) \\
\vdots & \vdots & \ddots & \vdots \\
\lambda(L-1) & \lambda(L-2) & \cdots & \lambda(0)
\end{array}\right]
$$

As a result, $S=\lambda(0)-\mathbf{V E}_{\mathbf{r}}^{-1} \mathbf{V}^{T}$ can be seen as the function of $L$.

Provided that $\lambda(0)$ equals one, that is, $\lambda(i)$ is the normalized correlation coefficient for generality,

$$
\begin{gathered}
\mathbf{E}_{\mathbf{r}}=\left[\begin{array}{cccc}
1 & \lambda(1) & \cdots & \lambda(L-1) \\
\lambda(1) & 1 & \cdots & \lambda(L-2) \\
\vdots & \vdots & \ddots & \vdots \\
\lambda(L-1) & \lambda(L-2) & \cdots & 1
\end{array}\right] \\
\mathbf{V}=[\lambda(L), \lambda(L-1), \cdots, \lambda(1)], \lambda(i)<1,0<i \leq L
\end{gathered}
$$

Therefore,

$$
S=1-\mathbf{V E}_{\mathbf{r}}^{-1} \mathbf{V}^{T}
$$

Consider the following relation,

$$
\mathbf{V}^{T} \mathbf{V}=\left[\begin{array}{cccc}
\lambda^{2}(L) & \lambda(L) \lambda(L-1) & \cdots & \lambda(L) \lambda(1) \\
\lambda(L-1) \lambda(L) & \lambda^{2}(L-1) & \cdots & \lambda(L-1) \lambda(1) \\
\vdots & \vdots & \ddots & \vdots \\
\lambda(1) \lambda(L) & \cdots & \cdots & \lambda^{2}(1)
\end{array}\right]
$$

In order to decide the signedness of $S^{-1}$, apply the matrix inversion lemma to Eq.40,

$$
S^{-1}=\left(1-\mathbf{V E}_{\mathbf{r}}^{-1} \mathbf{V}^{T}\right)^{-1}=1+\mathbf{V}\left(\mathbf{E}_{\mathbf{r}}-\mathbf{V}^{T} \mathbf{V}\right)^{-1} \mathbf{V}^{T}
$$

The positive matrix $\mathbf{E}_{\mathbf{r}}$ in Eq.(42) and the consideration of Eq.(41) result in the positive $\mathbf{E}_{\mathbf{r}}-\mathbf{V}^{T} \mathbf{V}$.

Hence $\mathbf{V}\left(\mathbf{E}_{\mathbf{r}}-\mathbf{V}^{T} \mathbf{V}\right)^{-1} \mathbf{V}^{T}$ is in the second order type and always bigger than 0 , leading to the fact that $S$ as well as $S^{-1}$ are bigger than 0 . Overall, $S$ is always positive and the inequality of Eq.(37) is always satisfied. As a result, Eq.(37) estimating the MMSE of the LMS algorithm in which the error signal is fed back monotonically decreases and stably converges according to the number of taps $L$. From Eq.(28) and the above result, it can be clearly seen that MMSE decreases when the number of taps in error feedback filter increases. 


\section{SIMULATION RESULTS}

In this section, the performance of the proposed DFE algorithm with L-taps error feedback filter is evaluated through MATLAB simulation by comparing it with the conventional DFE and LE. The channels used for the simulation is the simple ISI channel with additive white Gaussian noise (AWGN). The ISI channel model [1] used for the simulation is given as,

$$
h_{k}=\left\{\begin{array}{cc}
\frac{1}{2}\left[1+\cos \frac{2 \pi(k-1)}{K}\right], & k=0,1,2 \\
0, & \text { otherwise }
\end{array},\right.
$$

where, $K$ represents the parameter to adjust the degree of ISI.

The received signal $x_{k}$ is given as $x_{k}=d_{k} * h_{k}+n_{k}$ where $n_{k}$ is AWGN with variance $\sigma^{2}$ and $d_{k}$ is the BPSK-modulated signal. In the simulation, the number of taps for linear equalizer is 3 . For DFE, the numbers are 3 and 6 in the forward filter and the backward filter respectively. And the number of taps in the error feedback filter increases from 0 (for the conventional DFE) to 1 (for the DFE with one-tap error feedback filter [15]), 2, 3, 4 and 5 (for the proposed DFE). The step size for LMS algorithm is set to 0.01 .

In order to see more clearly the performance of each equalizer, the averaged values of ERLE (Echo Return Loss Enhancement) under various conditions are used in the simulation.

ERLE is defined as,

$$
E R L E=10 \log _{10}\left(\frac{E\left[d^{2}(n)\right]}{E\left[e^{2}(n)\right]}\right) .
$$

Figures from Fig.3(a) to Fig.3(d) represent the mean of ERLEs averaged with 5000 10000 iterations when MSE is converged, and compare the proposed method with previous methods.

From the Fig.3 and Fig.4, it can be explicitly seen that the DFE with error feedback filter outperforms the LE and DFE with respect to ELRE performance. It can also be seen that the ERLE of the proposed DFE algorithm is $0.65 \mathrm{~dB}$ on average better than that of the DFE with one-tap error feedback filter when the ISI characteristics parameter $K$ is 4.0 rather than 3.5, and the ERLE performance gets better when the channel variance $\sigma^{2}$ decreases. Especially when $K=4.0$, the increase of the number of taps to 2, 3 and 4 leads to nearly $0.22 \mathrm{~dB}$ improvement of the ERLE.

As a result, the proposed method significantly improves the ERLE performance compared to the DFE with one-tap error feedback filter when ISI is large and Gaussian noise is small, and the increase of the number of taps in the error feedback filter improves the ERLE performance, thus leading to the stable convergence of MMSE.

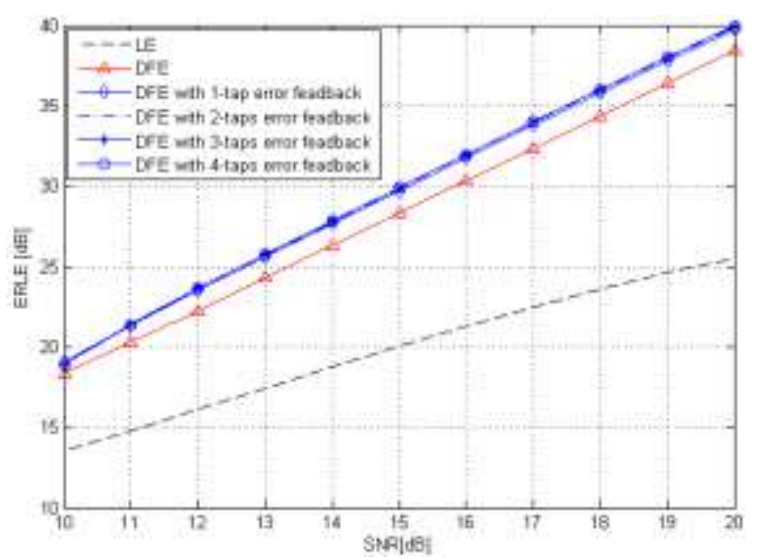

(a) $K=3.5, \sigma^{2}=0.01$

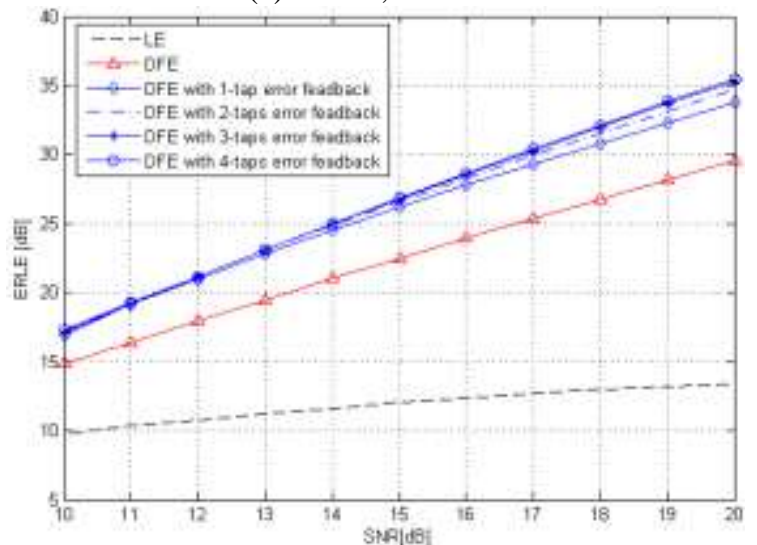

(b) $K=4, \sigma^{2}=0.01$

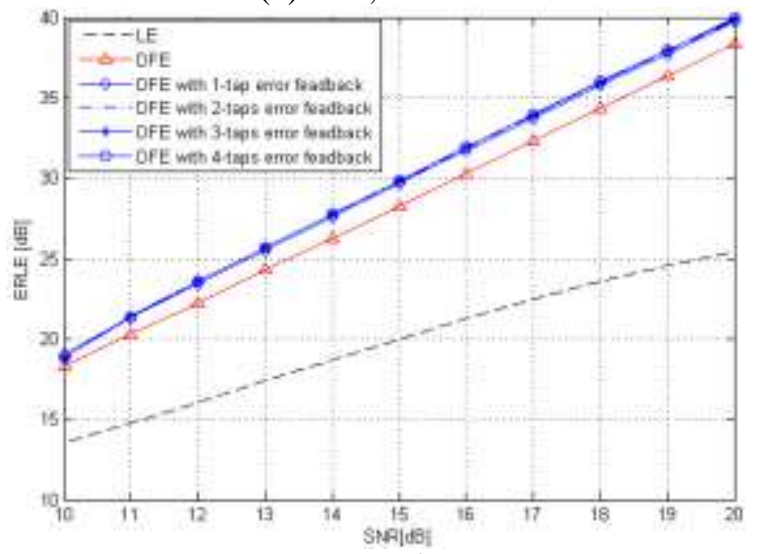

(c) $K=3.5, \sigma^{2}=0.03$

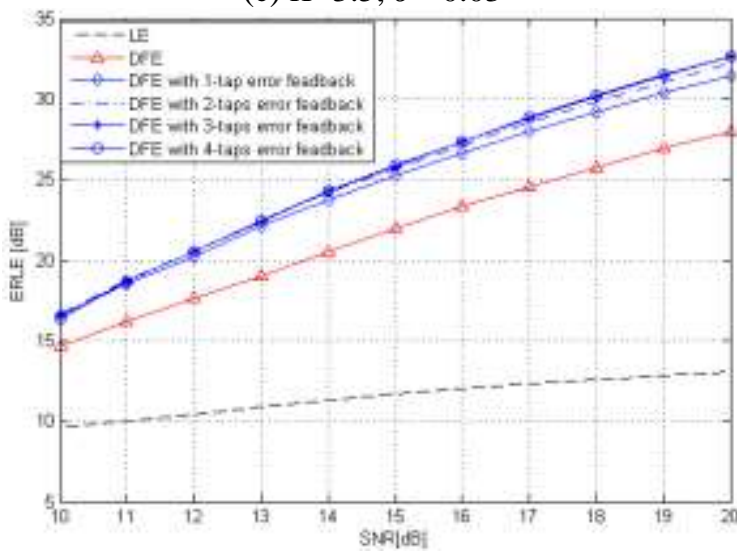

(d) $K=4, \sigma^{2}=0.03$

Fig.3. ELRE comparison $\left(\mathrm{K}=4, \sigma^{2}=0.03\right)$ 
The Fig.4 depicts the Bit Error Rate (BER) performance according to the number of taps. The Fig.4 clearly shows that the increase of the number of taps in the error feedback filter improves the BER performance in low SNR environments, especially when SNR below $15 \mathrm{~dB}$.

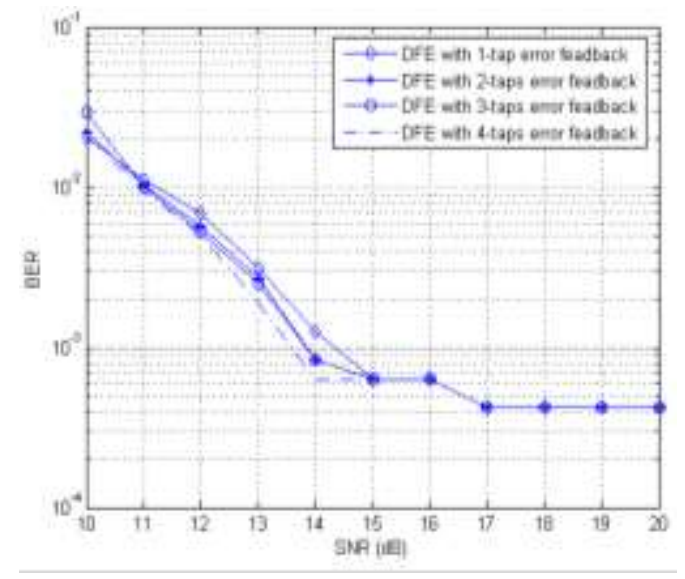

Fig.4. BER performance versus SNR for several algorithms

\section{CONCLUSIONS}

In this paper, the convergence and its stability of the generalized adaptive Decision Feedback Equalizer (DFE) algorithm with L-taps error feedback filter has been derived. The scheme is based on L-taps error feedback filter to reduce the correlation that exists in the error signal or to decrease the residual error variance that exists in the conventional DFE.

From the simulation results, it has been explicitly shown that the increase of number of taps in the error feedback filter improves the ERLE performance by $1.4 \mathrm{~dB}$ on average and convergence stability. In addition, it has been clearly seen that the DFE with several-taps error feedback filter significantly improves the BER performance, especially in low SNR environments.

For the proposed DFE with error feedback filter, the adaptive algorithm can be changed from LMS to other adaptive algorithms like RLS and the research on it is promising. Furthermore, the adaptive equalization processing is one kind of adaptive signal processing and can be utilized in the adaptive acoustic echo canceller. The adaptive acoustic echo canceller has the characteristics of great adaptation order and the exponential attenuation of the error signal, thus it will be our future research work by using the error feedback adaptive algorithm.

\section{REFERENCES}

[1] J.G. Proakis, "Digital Communications", McGraw-Hill, 2000.

[2] S. Haykin, “Adaptive Filter Theory”, Prentice-Hall, 1996.
[3] G.D. Forney, "Maximum-Likelihood Sequence Estimation of Digital Sequences in the Presence of Intersymbol Interference", IEEE Transactions on Information Theory, Vol. 18, No. 2, pp. 363-378, 1972.

[4] K. Okanoue, A. Ushirokawa, H. Tomita and Y. Furuya, "Fast Tracking Adaptive MLSE for TDMA Digital Cellular Systems", IEICE Transactions on Communications, Vol. 77, No. 5, pp. 557-564, 1994.

[5] K. Jamal, G. Brismark, and B. Gudmundson, "Adaptive MLSE Performance on the D-AMPS 1900 Channel”, IEEE Transactions on Vehicular Technology, Vol. 46, No. 3, pp. 73-75, 1997.

[6] J. F. Doherty and R.J. Mammone, "An Adaptive Algorithm for Stable Decision-Feedback Filtering”, IEEE Transactions on Circuits and Systems II: Analog and Digital Signal Processing, Vol. 40, No. 1, pp. 1-9, 1993.

[7] M. Rupp and A. Bahai, "Training and tracking of adaptive DFE algorithms under IS-136", Proceedings of $1^{\text {st }}$ IEEE Signal Processing Workshop Signal Processing Advances in Wireless Communications, pp. 341-344, 1997.

[8] R. Lopez-Valcarce, "Realizable Linear and Decision Feedback Equalizers: Properties and Connections", IEEE Transactions on Signal Processing, Vol. 52, No. 1, pp. $757-$ 773, 2004.

[9] D.L. Duttweiler, J.E. Mazo and D.G. Messerschmitt, “An Upper Bound on the Error Probability in Decision-Feedback Equalization", IEEE Transactions on Information Theory, Vol. 20, No. 4, pp. 490-497, 1974.

[10] J. Palicot, "A Weighted Decision Feedback Equalizer with Limited Error Propagation", Proceedings of International Conference on Communications, pp. 1-5, 2000.

[11] J. Palicot and Alban Goupil, "Performance Analysis of the Weighted Decision Feedback Equalizer", Signal Processing, Vol. 88, No. 2, pp. 284-295, 2008.

[12] T.I. Laakso and I.O. Hartimo, "Noise Reduction in Recursive Digital Filters using High-Order Error Feedback", IEEE Transactions on Signal Processing, Vol. 40, No. 5, pp. 1096-1106, 1992.

[13] S.K. Jagtap, M.S. Mulye and M.D. Uplane, "Speech Coding Techniques", Procedia Computer Science, Vol. 49, pp. 253263, 2015.

[14] Jagreeti Kaur and Baljit Singh, "Comparison of LSB and Predictive Coding using PSNR and MSE", International Journal of Computer Applications, Vol. 98, No. 7, pp. 1-12, 2014.

[15] Feng Wen Jiang, "Performance Analysis of a Decision Feedback Equalizer using Error Feedback", Information and Electronic Engineering, Vol. 4, No. 2, pp. 107-110, 2006.

[16] A.Y. Hassan, "A Wide Band QAM Receiver based on DFE to Reject ISI in Wireless Time Varying Fading Channel", International Journal of Electronics and Communications, Vol. 69, No. 1, pp. 332-343, 2015. 